\title{
17. Upon Stimulation of Human Hypothalamus
}

\author{
Keiji Sano, Masumi Yoshioka, Motohide Ogashrwa, \\ Buichi IsHrJima and Chihiro OHYE \\ Department of Neurosurgery, University of Tokyo
}

Destruction of the postero-medial par of the hypothalamus has been performed in order to calm or correct disorders of emotion as manifested in aggressive behaviors or rage attacks. This area is regarded as the ergotropic zone of the hypothalamus. The decision of the target point is done by two. menthods: one is under pneumoencephalographic control and the other is by observing the responses of the electrical stimulation of the proposed target area. The areas where rise in blood pressure, tachycardia, pupillary dilatation (bilateral, most often more marked on the ipsilateral side), and eye movements (various, often inward and downward movement of the ipsilateral eye), are elicited upon stimulation produced the calming effects remarkably and continuously by destruction, and this a rea is diffusely distributed in a zone. The region which produced markedly increase in blood pressure and tachycardia is found to be smaller in area than the region which produced pupillary dilatation, eye-ball movements and neck movements. In the lateral view, it is located in a triangle formed by the midpoint of the intercommissural line, the anterior border of the mammillary body and the rostral end of the aqueduct. And in the antero-posterior view, it is located from one to three $\mathrm{mm}$. lateral to the lateral wall of the third ventricle. This triangular zone is the ergotropic zone of the humun hypothalamus and may be called as the "ergotropic triangle".

The stimulation of the anterior or lateral part of the hypothalamus. yields most often parasympathetic responses. However, occassionally, no responses a re obtained. Upon stimulation of this triangle, desynchonization or in some cases irregular slowing of EEG and ECoG is noted in the neocortex and in the limbic structures except the region of the hippocampus. where often rhythmic theta waves are elicited. It was proved that two way connections exist in between the structures of the hippocampus, the amygdala and the postero-medial part of the hypothalamus as manifested by evoked potentials of low frequency stimulation.

Twenty cases of intractable violent behaviors (all having epileptic seizures) underwent the stereotactic surgery of postero-medial hypothalamotomy, all with satisfactory calming effects. 